\title{
Validation of a modified fluorimetric assay for the screening of trichomonacidal drugs
}

\author{
Alexandra Ibáñez Escribano ${ }^{1 /+}$, Alfredo Meneses Marcel $^{2}$, \\ Yanetsy Machado Tugores², Juan José Nogal Ruiz', Vicente J Arán Redó3, \\ José Antonio Escario García-Trevijano', Alicia Gómez Barrio'
}

\begin{abstract}
1'Departamento de Parasitologia, Facultad de Farmacia ${ }^{3}$ Instituto de Química Médica, Moncloa Campus of International Excellence, Universidad Complutense de Madrid, Universidad Politécnica de Madrid, Centro Superior de Investigaciones Centíficas, Madrid, España ${ }^{2}$ Department of Parasitology, Chemical Bioactive Center, Central University of Las Villas, Villa Clara, Cuba
\end{abstract}

\begin{abstract}
A fluorimetric microassay that uses a redox dye to determine the viability of the flagellate Trichomonas vaginalis has been optimised to provide a more sensitive method to evaluate potential trichomonacidal compounds. Resazurin has been used in recent years to test drugs against different parasites, including trichomonadid protozoa; however, the reproducibility of these resazurin-based methods in our laboratory has been limited because the flagellate culture medium spontaneously reduces the resazurin. The objective of this work was to refine the fluorimetric microassay method previously developed by other research groups to reduce the fluorescence background generated by the media and increase the sensitivity of the screening assay. The experimental conditions, time of incubation, resazurin concentration and media used in the microtitre plates were adjusted. Different drug sensitivity studies against $\mathrm{T}$. vaginalis were developed using the 5-nitroimidazole reference drugs, new 5-nitroindazolinones and 5-nitroindazole synthetic derivatives. Haemocytometer count results were compared with the resazurin assay using a 10\% solution of $3 \mathrm{mM}$ resazurin dissolved in phosphate buffered saline with glucose $(1 \mathrm{mg} / \mathrm{mL})$. The fluorimetric assay and the haemocytometer counts resulted in similar percentages of trichomonacidal activity in all the experiments, demonstrating that the fluorimetric microtitre assay has the necessary accuracy for high-throughput screening of new drugs against $\mathrm{T}$. vaginalis.
\end{abstract}

Key words: Trichomonas vaginalis - antitrichomonal agents - drug screening assay - resazurin - high-throughput screening assays

Trichomoniasis is one of the most common sexually transmitted diseases, with a prevalence of 180 million infections worldwide per year, which is higher than the number of reported cases of syphilis, chlamydia and gonorrhoea (Aggarwal \& Shier 2008). This cosmopolitan parasite can cause a wide range of clinical symptoms in the genitourinary tract, including vaginitis, cervicitis, endometritis and vulvitis in women and urethritis and prostatitis in men (Ali \& Nozaki 2007). However, the infection in males is generally asymptomatic and they are typically considered silent carriers of the disease (Cudmore et al. 2004).

Metronidazole and related 5-nitroimidazole derivatives are potent drugs used to treat infections caused by anaerobic or microaerophilic protozoa and bacteria, although resistance to these drugs has been demonstrated both in Trichomonas vaginalis isolates sampled from patients refractory to treatment (Wendel \& Workowski 2007, Aggarwal \& Shier 2008, Kissinger et al. 2008) and in laboratory-developed resistant isolates (Dunne et al. 2003). When the trichomonacide metronidazole was first introduced in 1959, its reported cure rate was approximately $95 \%$, but within two years of its introduction, the first

Financial support: AECID (A/030156/10), MICINN (SAF2009-10399), CEI-Campus Moncloa

+ Corresponding author: alexandraibanez@pdi.ucm.es

Received 21 December 2011

Accepted 19 March 2012 case of metronidazole resistance was reported in Canada (Lossick 1990). Clinical resistance has now been reported in most areas of the world (Dunne et al. 2003, Wendel \& Workowski 2007). Metronidazole is classified by the Food and Drug Administration as pregnancy category B, therefore some clinicians have used clotrimazole as an alternative treatment for trichomoniasis in pregnant women concerned about the possible foetal damaged caused by trichomoniasis (Swygard et al. 2004).

Recently, our group developed a quantitative structure-activity relationship model for the in silico prediction of new tricomonacidal products. However, experimental corroboration in vitro relies on the haemocytometer count (Marrero-Ponce et al. 2009), which is a laborious, time consuming, insensitive and subjective procedure.

Alamar Blue ${ }^{\circledR}$ has been used as a rapid, efficient and sensitive method for the antibacterial screening of new compounds against Mycobacterium tuberculosis, Bacillus spp and Staphylococcus spp (Barrow et al. 2007, Daryaee et al. 2009, Flemming et al. 2009), the determination of antifungal activity (Candida albicans, Aspergillus fumigatus) (Bowman et al. 2002, Repp et al. 2007), the screening of antiviral compounds (influenza virus) (Mo et al. 2008) and the screening of antiprotozoal drugs (T. vaginalis, Giardia duodenalis, Trypanosoma brucei, Leishmania spp, Acanthamoeba spp) (Raz et al. 1997, Mikus \& Steverding 2000, Campos Aldrete et al. 2005, McBride et al. 2005).

Alamar Blue ${ }^{\circledR}$ and its major component, resazurin, are blue and non-fluorescent dyes that become pink and fluorescent (resorufin) due to the chemical reduction of oxidoreductases present in viable cells (O'Brien et al. 2000). 
The fluorescence method with Alamar Blue ${ }^{\circledR}$ was first described by Campos Aldrete et al., in 2005. More recently, a resazurin-based method was described by Duarte et al. (2009b). We have been able to reproduce this resazurin-based method, but have observed interference between our culture media and the resazurin dye. Thus, we have modified the experimental conditions to increase the sensitivity of the method. Different drugs have been screened to validate the new protocol.

The aim of this study was the development and validation of a simple, rapid (one-step), sensitive and reliable method for use in our laboratory to screen new tricomonacidal drugs based on the fluorescence emitted by the redox indicator (resazurin) as it is reduced by viable protozoa. The results presented here enable the replacement of microscope-based methodologies with spectrofluorimetric assays.

\section{SUBJECTS, MATERIALS AND METHODS}

T. vaginalis - T. vaginalis (isolate $\mathrm{JH} 31 \mathrm{~A} \# 4$, ref. 30326; ATCC, MD, USA) was cultured in vitro in trypticose-yeast-maltose (TYM) media $(6.4 \mathrm{pH})$ supplemented with $10 \%(\mathrm{v} / \mathrm{v})$ heat-inactivated bovine serum. Cultures were incubated at $37^{\circ} \mathrm{C}$ in $5 \% \mathrm{CO}_{2}$ and subcultured every two-three days. Samples of the isolate were conserved in liquid $\mathrm{N}_{2}$ using TYM containing $10 \%$ heatinactivated foetal bovine serum and $10 \%$ of the cryoprotectant dimethyl sulfoxide (DMSO) (Sigma Chemical Co, St. Louis, MO).

Drugs - The drugs assayed during the method validation were the 5-nitroimidazoles metronidazole and secnidazole; clotrimazole, an antifungal compound used by some clinicians if the reference drugs, are contraindicated and new synthetic products. The compounds were dissolved in DMSO to measure their effectiveness against T. vaginalis via different methods. The final DMSO concentration in the culture media never exceeded $0.2 \%$ $(\mathrm{v} / \mathrm{v})$. Previous studies with $0.2 \%(\mathrm{v} / \mathrm{v})$ DMSO in $T$. vaginalis cultures were performed to confirm the absence of cytotoxic effects of the dissolvent in the cultures. All cultures (with and without DMSO) had the same growth rate (GR) (data not shown). The synthetic compound G-1 was previously evaluated by our laboratory (MenesesMarcel et al. 2008).

Resazurin preparation - Resazurin sodium salt (Sigma-Aldrich, St. Louis, MO, USA) was stored at $4^{\circ} \mathrm{C}$ and protected from light until use. Several resazurin stock solutions $(1,2,3$ and $4 \mathrm{mM})$ were prepared in $0.1 \%$ phosphate buffered saline/glucose $(\mathrm{PBS} / \mathrm{G})$ (Bénéré et al. 2007) and filter-sterilised prior to use. The reduction of resazurin by reductase activity was measured by fluorimetric readings taken in a plate fluorometer (Infinite 200, TECAN) at $\lambda_{\text {excitation }} 535 \mathrm{~nm}$ and $\lambda_{\text {emission }} 590 \mathrm{~nm}$ after performing the Alamar Blue ${ }^{\circledR}$ Assay (US Patent 5,501,959). Because carbohydrates are the main source of energy in T. vaginalis, glucose was added to the $\mathrm{PBS} / \mathrm{G}$ solution.

Assay optimisation - Before further optimisation, the spontaneous reduction of the resazurin in the culture media was demonstrated. Our results corroborate those described by other authors (Bénéré et al. 2007, Borra et al. 2009). TYM media without T. vaginalis and modified media lacking the reducing compounds ascorbic acid and L-cysteine were compared with PBS/G. The fluorescence of the plate was measured at different times and compared between these different media and nonresazurin media. The effect of the addition of $1 \mathrm{mg} / \mathrm{mL}$ glucose was also studied.

A similar experimental design was used after the addition of resazurin $(1,2,3$ and $4 \mathrm{mM} / 20 \mu \mathrm{L} /$ well $)$ with fluorimetric readings at regular time intervals to determine the optimal endpoint reading. A suspension of washed trophozoites (log-phase culture) was serially diluted two-fold in PBS in a 96-well microplate (Costar, NY, USA) $(200 \mu \mathrm{L} /$ well $)$ at a concentration between 6 x $10^{4}-4 \times 10^{6}$ trophozoites $/ \mathrm{mL}$. Then, the plates were incubated at $37^{\circ} \mathrm{C}\left(5 \% \mathrm{CO}_{2}\right)$ for $30 \mathrm{~min}, 1 \mathrm{~h}, 2 \mathrm{~h}, 3 \mathrm{~h}, 4 \mathrm{~h}$, $5 \mathrm{~h}$ and $6 \mathrm{~h}$. At each time point, a fluorescence intensity reading was performed for the experimental media and the PBS/G with resazurin control. The variation, correlation coefficients and other statistical parameters were calculated to adjust for the number of parasites, time of reading and resazurin concentration.

In vitro assay and experimental corroboration The in vitro activity of metronidazole, secnidazole, clotrimazole, G-1 and the synthetic derivatives of 5-nitroindazoles and 5-nitroindazolinones were compared via a microscope-based count in a Neubauer chamber and by the fluorescent method using resazurin. The tricomonacidal activity of metronidazole and secnidazole was studied at $0.0625,0.125,0.25,0.5,1$ and $2 \mu \mathrm{g} / \mathrm{mL}$. G-1 was studied at 5, 10 and $20 \mu \mathrm{g} / \mathrm{mL}$. Clotrimazole was studied at concentrations ranging from $0.625-10$ $\mu \mathrm{g} / \mathrm{mL}$ and the synthetic drugs were screened at 1,10 and $100 \mu \mathrm{g} / \mathrm{mL}$.

A logarithmic phase culture of $T$. vaginalis was seeded in $15 \times 150$-mm glass tubes. After 6-h incubation $(0 \mathrm{~h})$, the compounds were added to cultures containing $10^{5}$ protozoa $/ \mathrm{mL}$ in a final volume of $2 \mathrm{~mL}$ at the indicated concentrations. Three replicate experiments for each drug concentration were performed. A non-treated control $(100 \%$ growth) and culture medium control $(0 \%$ growth) were also used. After incubating for $24 \mathrm{~h}$ at $37^{\circ} \mathrm{C}$ in a humidified $5 \% \mathrm{CO}_{2}$ atmosphere, $200 \mu \mathrm{L}$ from each tube was seeded in sterile 96-well flat-bottomed microplates and centrifuged at $300 \mathrm{~g}$ for $5 \mathrm{~min}$ to eliminate the media. The parasites were resuspended in $200 \mu \mathrm{L}$ $\mathrm{PBS} / \mathrm{G}$ and $20 \mu \mathrm{L}$ resazurin (stock solution at $3 \mathrm{mM}$ ). After $1 \mathrm{~h}$ of incubation, the fluorescence was measured $\left(\lambda_{\text {ex }} 535 \mathrm{~nm}-\lambda_{\text {em }} 590 \mathrm{~nm}\right)$. The microscope-based haemocytometer count of viable flagellates was simultaneously assessed. At least three independent experiments were performed for each in vitro assay.

Expression of antiprotozoal activity - The percentage of growth inhibition (\% GI) was calculated over GR, which is the relation between the number of viable protozoa at $24 \mathrm{~h}$ and the number counted at $0 \mathrm{~h}$ (MuelasSerrano et al. 2000):

$$
\% \mathrm{GI}=[1-(\mathrm{GR} \text { drug/GR control) }] \times 100
$$




\section{RESULTS}

Spontaneous reduction of TYM media - A spontaneous reduction of resazurin and an accompanying increase in the fluorescence intensity of the TYM media used for the culture of T. vaginalis were observed (Fig. 1). Even TYM media without the reducing compounds L-cysteine and ascorbic acid (modified media) was fluorescent. The fluorescence units value recorded in the wells containing media was higher than those with viable parasites. The wells from which media had been removed had lower fluorescence than the wells containing trophozoites in PBS/G (Fig. 2). After taking these results into account, the protocol was changed for the subsequent experiments. Under the new protocol, after $24 \mathrm{~h}$ of drug exposure, $200 \mu \mathrm{L}$ of each culture was added into the wells and the media was removed before the addition of the resazurin solution. No fluorescence variation was noted at any resazurin concentration when using PBS/G $(1 \mathrm{mg} / \mathrm{mL})$ to replace the media. These results indicate that PBS is useful as an alternative, nonfluorescent medium.

Optimisation of the assay - Resazurin is an oxidationreduction dye that turns into a fluorescent compound (resorufin) after a reduction process caused by viable trophozoites. The intensity of fluorescence measured is proportional to parasite growth and can be quantified by fluorescence spectroscopy.

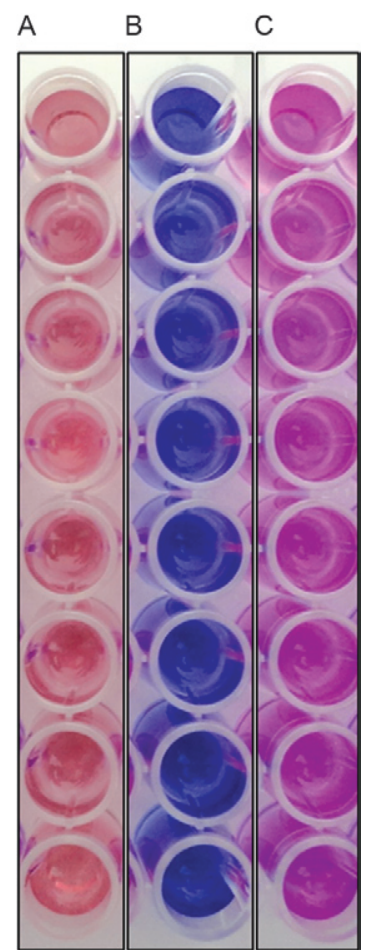

Fig. 1: comparison of colours in wells without trophozoites using trypticose-yeast-maltose (A) and phosphate buffered saline/glucose $(\mathrm{PBS} / \mathrm{G})(\mathrm{B})$ as medium after incubation with resazurin. C shows the pink resorufin colour obtained after incubation of Trichomonas vaginalis (100.000 trophozoites/mL) in PBS/G.
To optimise the method, the experimental parameters, such as the resazurin concentration, media used in the probe, inoculum of parasites and time of incubation with the redox dye, were adjusted.

Different concentrations of resazurin were tested, allowing us to obtain the smallest variation coefficient (Table I). The strongest linear correlation between the fluorescence percentage and protozoan density (Fig. 3) was noted at $3 \mathrm{mM}\left(\mathrm{R}^{2}=0.9916\right)$.

A standard curve for $T$. vaginalis at different incubation times with a $10 \%$ solution of $3 \mathrm{mM}$ resazurin is shown in Fig. 4. An optimum linearity was achieved after $1 \mathrm{~h}$ of incubation and the highest coefficient of linear determination $\left(\mathrm{R}^{2}=0.9983\right)$ was observed for $6 \times 10^{4}-4 \times$ $10^{6}$ trophozoites $/ \mathrm{mL}$.

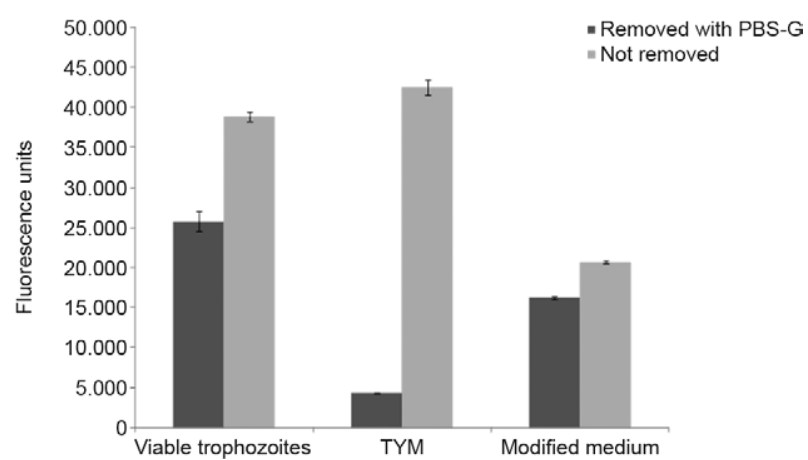

Fig. 2: evaluation of resazurin substrate reduction by different medium. Comparative fluorescence lectures of a Trichomonas vaginalis culture and fresh medium without trophozoites [complete trypticoseyeast-maltose (TYM) medium and modified medium without Lcysteine and ascorbic acid]. The fluorescence emitted was studied with prior removal with buffered saline/glucose (PBS/G) (dark bar) and without remove (gray bar). The fluorescence background obtained when the fresh medium without parasites is used in comparison with $\mathrm{PBS} / \mathrm{G}$ (2nd group of bars) revealing PBS/G as the most suitable for using with resazurin. All experiments were performed three times. Mean and standard deviation values are shown.

\section{TABLE I}

Determination of the resazurin concentration based on the coefficient variation of fluorescence values of Trichomonas vaginalis cultures at different time incubation

Time

\begin{tabular}{lcccc} 
(h) & $4 \mathrm{mM}$ & $3 \mathrm{mM}$ & $2 \mathrm{mM}$ & $1 \mathrm{mM}$ \\
\hline 0.5 & 10.16 & 4.82 & 7.20 & 7.55 \\
1 & 5.28 & 3.06 & 2.60 & 3.20 \\
2 & 3.83 & 1.61 & 1.59 & 2.46 \\
3 & 2.86 & 1.53 & 1.47 & 2.29 \\
4 & 2.32 & 1.21 & 1.37 & 2.03 \\
5 & 2.20 & 1.05 & 1.41 & 2.00 \\
6 & 2.14 & 1.07 & 1.31 & 1.96 \\
Mean \pm SD & $4.11 \pm 2.9$ & $2.05 \pm 1.4$ & $2.42 \pm 2.2$ & $3.07 \pm 2.0$
\end{tabular}

SD: standard deviation. 
In vitro drug susceptibility assay - To determine the reliability of this fluorescence method for the screening of new products against $T$. vaginalis, reference drugs, alternative drugs for trichomoniasis treatment (metronidazole, secnidazole and clotrimazole) and synthetic

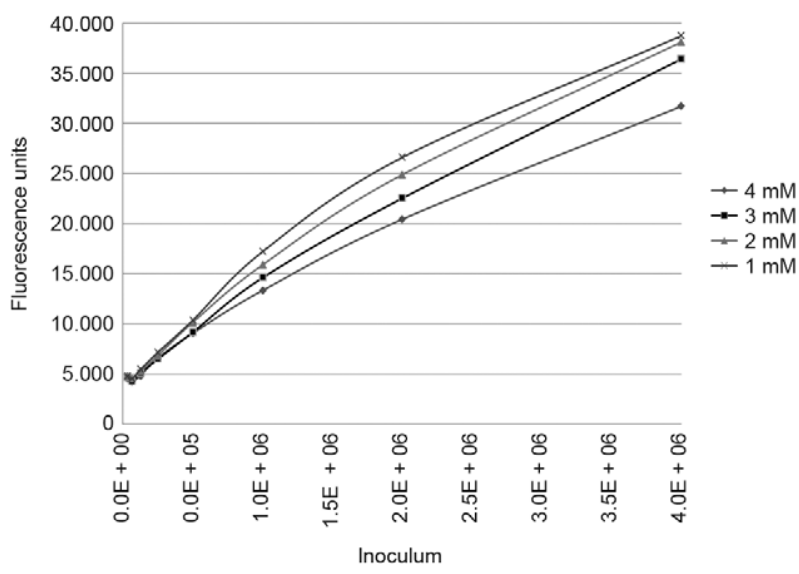

Fig. 3: change in resazurin reduction with dye concentration and plating density of Trichomonas vaginalis after $1 \mathrm{~h}$ of incubation time with $20 \mu \mathrm{L}$ of different resazurin solution. The concentrations studied were $1 \mathrm{mM}, 2 \mathrm{mM}, 3 \mathrm{mM}$ and $4 \mathrm{mM}$ showing a correlation coefficient of $0.9728,0.9841,0.9916$ and 0.9728 respectively. The optimal value $\left(\mathrm{R}^{2}\right.$ $=0.9916)$ is shown at $3 \mathrm{mM}$ concentration. Each signal is the average of six individual experiments.

A

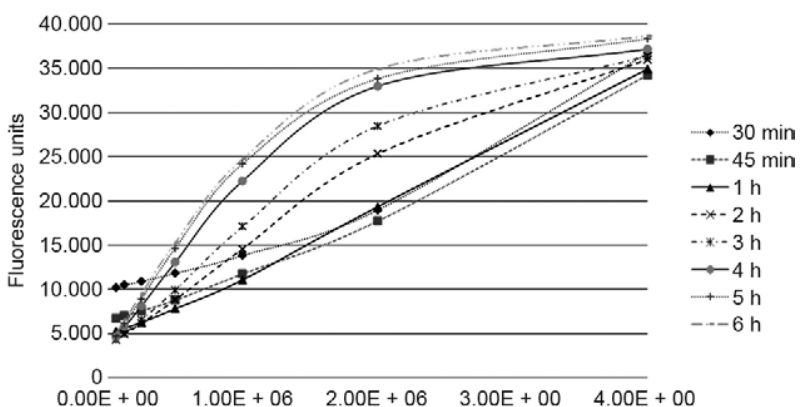

B

\begin{tabular}{cccc}
\multicolumn{4}{c}{ T. vaginalis $/ \mathrm{mL}$} \\
\hline Time & Slope & Intercept & $\mathrm{R}^{2}$ \\
\hline $30 \mathrm{~min}$ & 0.0066 & 8680.8 & 0.9713 \\
$45 \mathrm{~min}$ & 0.0069 & 8561.1 & 0.9910 \\
1h & $\mathbf{0 . 0 0 7 6}$ & $\mathbf{4 2 6 8}$ & $\mathbf{0 . 9 9 8 3}$ \\
2h & 0.0083 & 4861.4 & 0.9728 \\
3h & 0.0085 & 5755.4 & 0.9359 \\
4h & 0.0086 & 7960.3 & 0.8574 \\
5h & 0.0087 & 8784.1 & 0.8440 \\
6h & 0.0087 & 9340.2 & 0.8334 \\
\hline
\end{tabular}

Fig. 4: correlation between resazurin reduction $(3 \mathrm{mM})$ and the number of Trichomonas per millilitre after different incubation periods. A: graphic representation of standard curves of Trichomonas vaginalis cultures at different incubation times; B: correlation data between the different standard curves. The time of incubation with best correlation $\left(\mathrm{R}^{2}=0.9983\right)$ between fluorescent and viable trophozoites has been $1 \mathrm{~h}$. compounds (G-1, 5-nitroindazole and 5-nitroindazolinone derivatives) were tested using the resazurin assay and the microscope-based counting method simultaneously. No relevant difference between the \% GI was observed when comparing both methods (Tables II, III). An analysis of variance (ANOVA) was performed using SPSS software (v.20, IBM) on the data collected using both methods. There was no statistically significant difference $(p>0.05)$ between the resazurin and the microscope-based assays. The quality of the resazurinbased method presented here for use in high-throughput screening assays has been estimated using the $Z^{\prime}$-factor (Zhang et al. 1999). The obtained result $\left[Z^{\prime}=0.8(1>\right.$ $\left.Z^{\prime} \geq 0.5\right)$ ] suggests that the resazurin assay has excellent potential for screening.

Each plate should include control wells without parasites to avoid false negative results that could occur if any of the assayed products reduce the resazurin or produce fluorescence. In addition, growth and metronidazole control wells should be included.

\section{DISCUSSION}

Our results demonstrate that the resazurin-based fluorimetric method is a fast, non-subjective, reliable, sensitive and affordable assay for in vitro drug screening against the human protozoan $T$. vaginalis.

\section{TABLE II}

Percentage of fluorescence inhibition (\% FI) and percentage of growth inhibition ( $\%$ GI) of therapeutic drugs determined by resazurin and microscopic counting against Trichomonas vaginalis

\begin{tabular}{lccc}
\hline $\begin{array}{l}\text { Therapeutic } \\
\text { drugs }\end{array}$ & $\begin{array}{c}\text { Concentration } \\
\mu \mathrm{g} / \mathrm{mL}\end{array}$ & $\begin{array}{c}\% \mathrm{FI} \\
\text { resazurin }^{a}\end{array}$ & $\begin{array}{c}\% \mathrm{GI} \\
\text { haemocytometer }\end{array}$ \\
\hline Metronidazole & 2 & $100 \pm 0$ & $94.86 \pm 0.03$ \\
& 1 & $92.1 \pm 5.58$ & $91.5 \pm 2.77$ \\
& 0.5 & $77.6 \pm 4.14$ & $81.06 \pm 4.14$ \\
& 0.25 & $46.15 \pm 9.36$ & $51.64 \pm 9.36$ \\
& 0.125 & $20.16 \pm 8.45$ & $19.9 \pm 8.45$ \\
Secnidazole & 0.0625 & $13.96 \pm 7.08$ & $3.78 \pm 7.08$ \\
& 2 & $99.95 \pm 0.03$ & $100 \pm 0$ \\
& 1 & $92.07 \pm 0.67$ & $95 \pm 1.7$ \\
& 0.5 & $75.37 \pm 8.46$ & $79.71 \pm 8.3$ \\
& 0.25 & $48.8 \pm 13.72$ & $55.14 \pm 8.08$ \\
& 0.125 & $30.65 \pm 0.96$ & $45.71 \pm 10.49$ \\
& 0.0625 & $31.17 \pm 1.09$ & $30 \pm 12.63$ \\
& 10 & $100 \pm 0$ & $84.63 \pm 2.01$ \\
& 5 & $79.32 \pm 6.91$ & $75.53 \pm 2.16$ \\
& 2.5 & $54.86 \pm 16.95$ & $65.54 \pm 5.23$ \\
& 1.25 & $41.49 \pm 3.86$ & $51.74 \pm 6.73$ \\
& 0.625 & $36.5 \pm 8.59$ & $21.49 \pm 8.43$
\end{tabular}

$a$ : the results obtained show no difference between methodologies $(\mathrm{p}>0.05)$. All experiments are performed at least per triplicate. Statistical comparisons of both methods were performed by the analysis of variance test with SPSS software (v.20, IBM). 
The traditional method used for testing new compounds against this flagellate is based on microscopebased counting of viable trophozoites with a haemocytometer (Muelas-Serrano et al. 2000, Gomez Barrio et al. 2004, Tiwari et al. 2008). This technique has several drawbacks: it is time consuming, laborious and a highly dependent on the observer. Several drug sensitivity assays for this parasite have been described using radiolabelled nucleotides (Adagu et al. 2002) or colourimetric assays utilising $p$-nitrophenyl phosphate (Martinez-Grueiro et al. 2003) or tetrazolium salts (Zhang et al.
2009) as chromogenic substrates. These screening tests have been proposed as alternatives to microscope-based counting; however, the fluorescent and colorimetric dye Alamar Blue ${ }^{\circledR}$ permits the development of a non-radioactive, sensitive and one-step assay (Raz et al. 1997). In the years following the development of the fluorescent technique, resazurin, which is the major constituent of the Alamar Blue ${ }^{\circledR}$ started to be used in many assays due to lower cost. Resazurin is currently being used instead of Alamar Blue ${ }^{\circledR}$ to evaluate natural and synthetic products against bacteria, fungi and parasites (Rolon et al.

TABLE III

Trichomonacidal activity of synthetic products (G-1, 5-nitroindazoles, 5-nitraindazolinones derivatives) by resazurin and haemocytometer methods

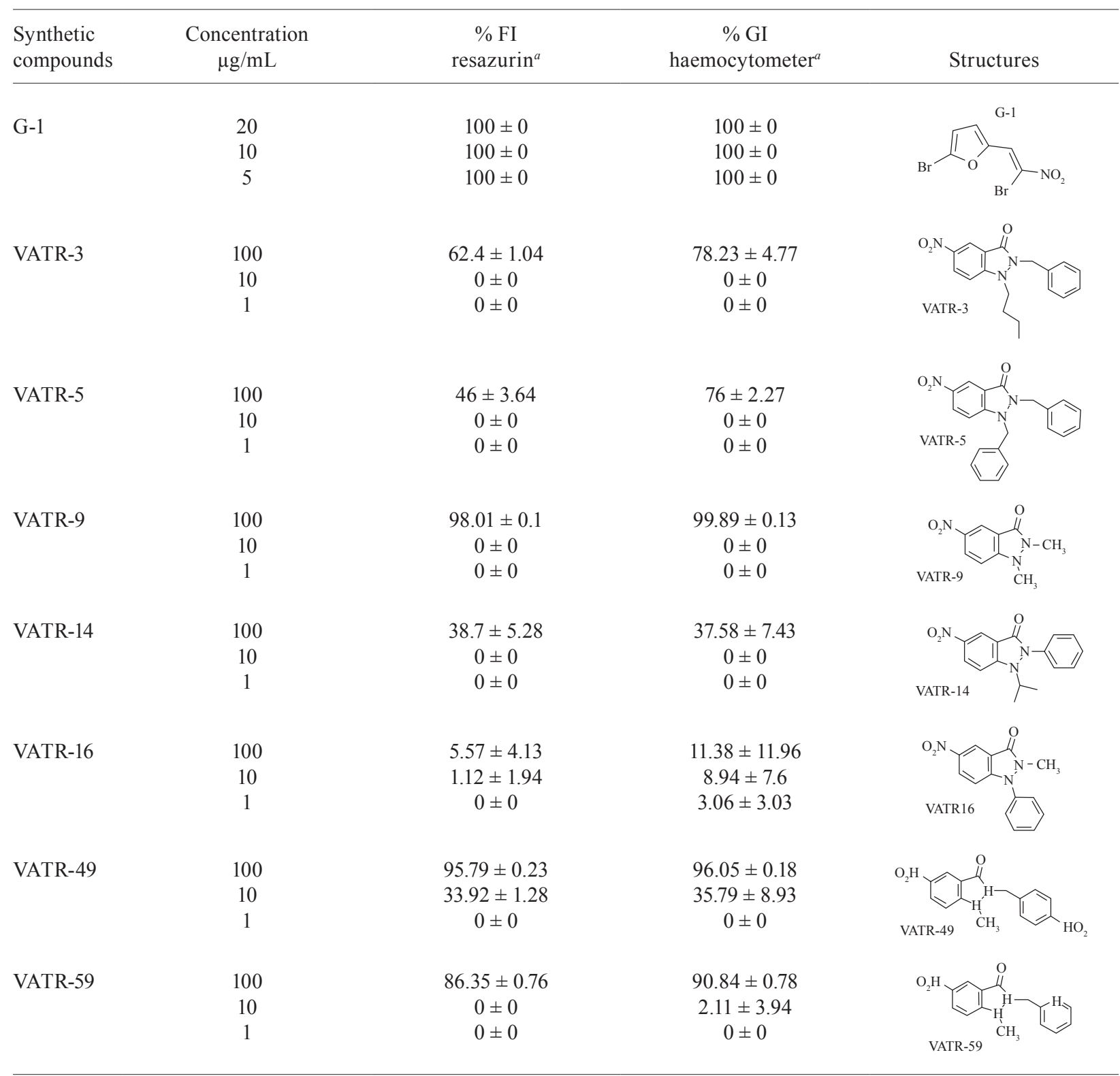

$a$ : the results obtained show no difference between methodologies ( $\mathrm{p}>0.05) ; \%$ FI: fluorescence inhibition; \% GI: percentage of growth inhibition. 
2006, Bénéré et al. 2007, Sarker et al. 2007, Duarte et al. 2009a, b, Mirza et al. 2011) and to study the proliferation of mammalian cells (O'Brien et al. 2000). A fluorescence screening method for $T$. vaginalis has already been developed (Campos Aldrete et al. 2005, Duarte et al. 2009b). The main differences between the different fluorescent assays are the number of trophozoites per well, the excitation and emission wavelength for the fluorimetric determination and the choice of redox indicator. Although Campos Aldrete et al. (2005) developed a screening test using Alamar Blue ${ }^{\circledR}$, Duarte et al. (2009b) used the more affordable resazurin dye. In both cases, the media used for the culture of the trophozoites is not removed.

In accordance with the results of a 2007 study by Bénéré et al. and a 2009 study by Borra et al., our group observed a spontaneous reduction of resazurin by trichomonadid culture media (Fig. 1). These results suggest that one or more compounds in the TYM medium reduce the fluorescent substrate. A modified medium lacking L-cysteine and ascorbic acid was studied, revealing the reductive behaviour of both components, though at a lower reduction percentage than the complete fresh media. As shown in Fig. 2, media lacking parasites had higher fluorescence than the complete fresh media with trophozoites. This effect is likely the result of the consumption of nutrients in the TYM media by viable flagellates, resulting in the reduction of resazurin. The reductive effect of yeast extract was not confirmed, because media without this component would not have sufficient nutrients for the correct maintenance and growth of the isolate. Yeast extract and vitamins are present in the different trichomonadid culture media (Petrin et al. 1998) used by different research groups (Campos Aldrete et al. 2005, Duarte et al. 2009b). Our study presents a modified protocol that removes the reductive media, guaranteeing that resorufin can only be generated by the metabolic activity of viable $T$. vaginalis. This, in turn, lowers the possibility of false negatives caused by spontaneous media-related reduction.

Standard curves for $T$. vaginalis (Figs 3, 4) indicate a correlation between the fluorescence emitted by resorufin and the quantity of viable flagellates in the logarithmic growth phase. These curves allowed the direct in vitro determination of the trichomonacidal effect of different compounds added to the parasite cultures. The addition of $1 \mathrm{mg} / \mathrm{mL}$ glucose did not have any marked influence on the reduction of resazurin (data not shown) as was observed with other research groups (Bénéré et al. 2007). Different 5-nitroimidazole drugs, the antifungal clotrimazole and synthetic products were tested to confirm the effectiveness of this modified assay. The results obtained by both screening procedures (microscopebased counting vs. fluorimetric assay) corroborate the reliability of this resazurin-based assay (Tables II, III). The comparison of the population average of the two methodologies with the ANOVA test also indicated no divergence between results. Although resazurin is a fluorimetric and colourimetric redox indicator, this research was developed using fluorescence measurements. Previous studies indicate that fluorescence readings are more sensitive than spectrophotometry (O'Brien et al. 2000).
Overall, we conclude that the media used for the in vitro culture of $T$. vaginalis can reduce the fluorescent dye resazurin, thus increasing the fluorescence background of the probe. Therefore, it is essential to remove the culture media from the wells to increase the accuracy of the assay. The modified fluorimetric method described in this study could be easily automated for high-throughput screening.

\section{REFERENCES}

Adagu IS, Nolder D, Warhurst DC, Rossignol JF 2002. In vitro activity of nitazoxanide and related compounds against strains of Giardia intestinalis, Entamoeba histolytica and Trichomonas vaginalis. J Antimicrob Chemoth 49: 103-111.

Aggarwal A, Shier RM 2008. Recalcitrant Trichomonas vaginalis infections successfully treated with vaginal acidification. J Obstet Gynaecol Can 30: 55-58.

Ali V, Nozaki T 2007. Current therapeutics, their problems and sulfur-containing-amino-acid metabolism as a novel target against infections by "amitochondriate" protozoan parasites. Clin Microbiol Rev 20: 164-187.

Barrow EW, Dreier J, Reinelt S, Bourne PC, Barrow WW 2007. In vitro efficacy of new antifolates against trimethoprim-resistant $B a$ cillus anthracis. Antimicrob Agents Chemother 51: 4447-4452.

Bénéré E, da Luz RA, Vermeersch M, Cos P, Maes L 2007. A new quantitative in vitro microculture method for Giardia duodenalis trophozoites. J Microbiol Methods 71: 101-106.

Borra RC, Lotufo MA, Gagioti SM, Barros Fde M, Andrade PM 2009. A simple method to measure cell viability in proliferation and cytotoxicity assays. Braz Oral Res 23: 255-262.

Bowman JC, Hicks PS, Kurtz MB, Rosen H, Schmatz DM, Liberator PA, Douglas CM 2002. The antifungal echinocandin caspofungin acetate kills growing cells of Aspergillus fumigatus in vitro. Antimicrob Agents Chemother 46: 3001-3012.

Campos Aldrete ME, Salgado-Zamora H, Luna J, Melendez E, MerazRios, MA 2005. A high-throughput colorimetric and fluorometric microassay for the evaluation of nitroimidazole derivatives anti-trichomonas activity. Toxicol in Vitro 19: 1045-1050.

Cudmore SL, Delgaty, KL, Hayward-McClell SF, Petrin DP, Garber GE 2004. Treatment of infections caused by metronidazole-resistant Trichomonas vaginalis. Clin Microbiol Rev 17: 783-793.

Daryaee F, Kobarfard F, Khalaj A, Farnia P 2009. Synthesis and evaluation of in vitro anti-tuberculosis activity of $\mathrm{N}$-substituted glycolamides. Eur J Med Chem 44: 289-295.

Duarte M, Giordani RB, Carli GA, Zuanazzi JA, Macedo AJ, Tasca T 2009a. Cytotoxicity of solubilization vehicles for Trichomonas gallinae and Tritrichomonas foetus measured by the resazurin microtiter assay. Vet Parasitol 166: 167-170.

Duarte M, Giordani RB, De Carli GA, Zuanazzi JA, Macedo AJ, Tasca T 2009b. A quantitative resazurin assay to determinate the viability of Trichomonas vaginalis and the cytotoxicity of organic solvents and surfactant agents. Exp Parasitol 123: 195-198.

Dunne RL, Dunn LA, Upcroft P, O’Donoghue PJ, Upcroft JA 2003. Drug resistance in the sexually transmitted protozoan Trichomonas vaginalis. Cell Res 13: 239-249.

Flemming K, Klingenberg C, Cavanagh JP, Sletteng M, Stensen W, Svendsen JS, Flaegstad T 2009. High in vitro antimicrobial activity of synthetic antimicrobial peptidomimetics against staphylococcal biofilms. J Antimicrob Chemother 63: 136-145.

Gomez Barrio A, Martinez Grueiro MM, Montero D, Nogal JJ, Escario JA, Muelas S, Fernandez C, Vega C, Rolon M, Martinez 
Fernandez AR, Solis PN, Gupta MP 2004. In vitro antiparasitic activity of plant extracts from Panama. Pharm Biol 42: 332-337.

Kissinger P, Secor WE, Leichliter JS, Clark RA, Schmidt N, Curtin E, Martin DH 2008. Early repeated infections with Trichomonas vaginalis among HIV-positive and HIV-negative women. Clin Infect Dis 46: 994-999.

Lossick JG 1990. Treatment of urogenital trichomoniasis. In BM Honigberg, Trichomonads parasitic in human, Springer Verlag, New York, p. 324-341.

Marrero-Ponce Y, Rivera-Borroto OM, Meneses-Marcel A, Escario JA, Gomez Barrio A, Aran VJ, Alho MAM, Pereira DM, Nogal JJ, Torrens F, Ibarra-Velarde F, Montenegro YV, Huesca-Guillen A, Rivera N, Vogel C 2009. Discovery of novel trichomonacidals using LDA-Driven QSAR models and bond-based bilinear indices as molecular descriptors. Qsar Comb Sci 28: 9-26.

Martinez-Grueiro MM, Montero-Pereira D, Gimenez-Pardo C, Nogal-Ruiz JJ, Escario JA, Gomez-Barrio A 2003. Trichomonas vaginalis: determination of acid phosphatase activity as a pharmacological screening procedure. J Parasitol 89: 1076-1077.

McBride J, Ingram PR, Henriquez FL, Roberts CW 2005. Development of colorimetric microtiter plate assay for assessment of antimicrobials against Acanthamoeba. J Clin Microbiol 43: 629-634.

Meneses-Marcel A, Rivera-Borroto OM, Marrero-Ponce Y, Montero A, Machado Tugores Y, Escario JA, Gomez Barrio A, Montero Pereira D, Nogal JJ, Kouznetsov VV, Ochoa Puentes C, Bohorquez AR, Grau R, Torrens F, Ibarra-Velarde F, Aran VJ 2008. New antitrichomonal drug-like chemicals selected by bond (edge)-based TOMOCOMD-CARDD descriptors. J Biomol Screen 13: 785-794.

Mikus J, Steverding D 2000. A simple colorimetric method to screen drug cytotoxicity against Leishmania using the dye Alamar Blue. Parasitol Int 48: 265-269.

Mirza H, Teo JD, Upcroft J, Tan KS 2011. A rapid, high-throughput viability assay for Blastocystis spp reveals metronidazole resistance and extensive subtype-dependent variations in drug susceptibilities. Antimicrob Agents Chemother 55: 637-648.

Mo C, Yamagata R, Pan A, Reddy J, Hazari N, Duke G 2008. Development of a high-throughput Alamar Blue assay for the determination of influenza virus infectious dose, serum antivirus neutralization titer and virus ca/ts phenotype. J Virol Methods 150: 63-69.
Muelas-Serrano S, Nogal J, Martínez-Díaz RA, Escario JA, Martínez-Fernández R, Gómez-Barrio A 2000. In vitro screening of American plant extracts on Trypanosoma cruzi and Trichomonas vaginalis. J Ethnopharmacol 71: 101-107.

O’Brien J, Wilson I, Orton T, Pognan F 2000. Investigation of the Alamar Blue (resazurin) fluorescent dye for the assessment of mammalian cell cytotoxicity. Eur J Biochem 267: 5421-5426.

Petrin D, Delgaty K, Bhatt R, Garber G 1998. Clinical and microbiological aspects of Trichomonas vaginalis. Clin Microbiol Rev 11: 300-317.

Raz B, Iten M, Grether-Buhler Y, Kaminsky R, Brun R 1997. The Alamar Blue assay to determine drug sensitivity of African trypanosomes (T.b. rhodesiense and T.b. gambiense) in vitro. Acto Trop 68: 139-147.

Repp KK, Menor SA, Pettit RK 2007. Microplate Alamar Blue assay for susceptibility testing of Candida albicans biofilms. Med Mycol 45: 603-607.

Rolon M, Vega C, Escario JA, Gomez-Barrio A 2006. Development of resazurin microtiter assay for drug sensibility testing of Trypanosoma cruzi epimastigotes. Parasitol Res 99: 103-107.

Sarker SD, Nahar L, Kumarasamy Y 2007. Microtitre plate-based antibacterial assay incorporating resazurin as an indicator of cell growth and its application in the in vitro antibacterial screening of phytochemicals. Methods 42: 321-324.

Swygard H, Sena AC, Hobbs MM, Cohen MS 2004. Trichomoniasis: clinical manifestations, diagnosis and management. Sex Transm Infect 80: 91-95.

Tiwari P, Singh D, Singh MM 2008. Anti-trichomonas activity of Sapindus saponins, a candidate for development as microbicidal contraceptive. J Antimicrob Chemother 62: 526-534.

Wendel KA, Workowski KA 2007. Trichomoniasis: challenges to appropriate management. Clin Infect Dis 44: S123-S129.

Zhang J, Chung TDY, Oldenburg KR 1999. A simple statistical parameter for use in evaluation and validation of high throughput screening assays. J Biomol Screen 4: 67-73.

Zhang XX, Zheng SS, Zhu MM 2009. In vitro effect of secnidazole benzoate on Trichomonas vaginalis. Chinese Journal of Parasitology \& Parasitic Diseases 27: 111-114. 